\title{
The Influence of Mecamylamine on Trigeminal and Olfactory Chemoreception of Nicotine
}

\author{
Norbert Thuerauf*,', Katrin Markovic', Georg Braun', Stefan Bleich', Udo Reulbach', Johannes Kornhuber' \\ and Jens Lunkenheimer' \\ 'Sensory Research Unit, Department of Psychiatry, University of Erlangen-Nuremberg, Erlangen, Bavaria, Germany
}

\begin{abstract}
Nicotine presented to the nasal cavity at low concentrations evokes 'odorous' sensations, and at higher concentrations 'burning' and 'stinging' sensations. A study in smokers and nonsmokers provided evidence of a relationship between the experience with the pharmacological action of S-(-)-nicotine and the perceived pleasantness/unpleasantness following nasal stimulation with S-(-)-nicotine. Mecamylamine, a nicotinic acetylcholine-receptor-(nAch-R) antagonist, was able to block painful responses following chemical stimulation of the human tongue and to block responses from the rat's ethmoidal nerve. The aim of our study in humans was to investigate the effects of mecamylamine on the olfactory and the trigeminal chemoreception of nicotine enantiomers. In order to achieve this aim, we determined-before and after mecamylamine-(I) detection thresholds, trigeminal thresholds, and intensity estimates (stimulus intensity) and (2) recorded the negative mucosal potential (NMP) following nasal stimulation with nicotine in a placebo-controlled double blind study $(n=15) . \mathrm{CO}_{2}$ was used as a trigeminal and $\mathrm{H}_{2} \mathrm{~S}$ as an olfactory control stimulus. Mecamylamine significantly increased trigeminal thresholds of $\mathrm{S}-(-)$-nicotine and reduced intensity estimates and NMPs following stimulation with nicotine enantiomers, whereas mecamylamine did not influence NMPs and trigeminal intensity estimates following stimulation with $\mathrm{CO}_{2}$. In contrast, mecamylamine did neither influence detection thresholds nor olfactory intensity estimates following stimulation with olfactory nicotine concentrations. These results demonstrate that the trigeminal nasal chemoreception of nicotine enantiomers, in contrast to $\mathrm{CO}_{2}$, is mediated by $\mathrm{nAch}$-Receptors and give evidence that the olfactory chemoreception of nicotine is independent from peripheral $\mathrm{nAch}$ Receptors.
\end{abstract}

Neuropsychopharmacology (2006) 3 I, 450-46I. doi:I0.1038/sj.npp. I 300842; published online 3 August 2005

Keywords: nicotine; chemoreception; mecamylamine; olfaction; smell; pain

\section{INTRODUCTION}

Nicotine not only represents the main addictive component of smoking but it also greatly contributes to the sensory properties of tobacco (Laviolette and van der Kooy, 2004; Walker et al, 1996; Hummel et al, 1992a, b; Thuerauf et al, $1999,2000)$. The substance presented to the nasal cavity at low concentrations evokes 'odorous' sensations and at higher concentrations 'burning' and 'stinging' sensations, possibly mediated by nicotinic acetylcholine receptors (nAchR) (Walker et al, 1996; Thuerauf et al, 1999). In contrast to the majority of other chemical compounds, nicotine activates the olfactory and the trigeminal system at

\footnotetext{
*Correspondence: Dr N Thuerauf, Klinik mit Poliklinik für Psychiatrie und Psychotherapie, University of Erlangen-Nuremberg, Schwabachanlage 6, Erlangen 91054, Germany, Tel: +49 (0) 9131 8534892 or 8534807, Fax: + 49 (0) 9131 8534806,

E-mail: norbert.thuerauf@rzmail.uni-erlangen.de

Received 22 February 2005; revised 31 May 2005; accepted 17 June 2005

Online publication: 21 June 2005 at http://www.acnp.org/citations/ Npp062 105050 I25/default.pdf
}

relatively low concentrations (Hummel et al, 1992a,b). During the last decade stereospecific differences between nicotine enantiomers have been reported for the nasal trigeminal sensory system (Thuerauf et al, 1999, 2000; Walker et al, 1996). S-(-)-nicotine - the natural isomer of tobacco - possesses lower trigeminal thresholds and higher stimulus strength for the 'burning' and the 'stinging' sensation compared to $R-(+)$-nicotine. Smokers experience the sensory properties of $S-(-)$-nicotine during smoking but both-smokers and nonsmokers-are naïve to the sensory properties of $R-(+)$-nicotine. Two studies in smokers and nonsmokers provided evidence of a relationship between the experience with the pharmacological action of $S-(-)$-nicotine and the hedonic rating (pleasantness/unpleasantness) (Hummel et al, 1992a; Thuerauf et al, 2000). Smokers perceived the $S$-(-)-isomer as being more pleasant (or less unpleasant) than nonsmokers. The difference in hedonic estimates has been explained by smokers' experience with the actions of $S$-(-)-nicotine pointing strongly to a conditioning mechanism between the sensory stimulus and the pleasant pharmacological actions of $S-(-)$-nicotine. In one of the two studies in smokers and 
nonsmokers, nitrogen was used as carrier gas for nicotine enantiomers and precise concentration measurements by means of a UV-detection method were conducted (Thuerauf et al, 2000). This study clearly revealed (1) that the different hedonic estimates of smokers and nonsmokers reflect hedonic differences in nicotine stereoisomers and not differences due to compounds formed by degradation and (2) that the differences in hedonic estimates appear exclusively when nicotine stimulus concentrations that activate the trigeminal system were used. It is well known that these stimulus concentrations are achieved during the process of cigarette smoking (Davies and Vaught, 1990; Thuerauf et al, 2000).

Recently, we demonstrated in humans the existence of specific stereoselective receptors for the nasal trigeminal system by the stereoselective dose-dependent activation with highly purified $R(+)$ - and $S(-)$-nicotine (Thuerauf et al, 1999). In this study, trigeminal activation was measured by recording negative mucosal potentials (NMPs) from the human nasal mucosa. Whereas responses from single trigeminal primary afferents in the nasal mucosa cannot be recorded in humans, a summated pain-related electrical response - the NMP - can (Kobal, 1985). Nonpainful chemical stimuli were not able to elicit NMPs. In animal experiments we demonstrated the sensitivity of NMPs to capsaicin pretreatment and the independence of NMPs from autonomic reflexes (Thuerauf et al, 1991, 1993). Furthermore, it was shown that NMPs are restricted to the site of stimulation, that is, to the area of activated nociceptors (Thuerauf et al, 1993). Summarizing these investigations so far, the NMP can be characterized as a specific peripherally nociceptive correlate suitable for quantifying trigeminal activation.

Recently, the technique of NMP recording has been improved by positioning the recording electrode under endoscopic control (Hummel et al, 1996). This technique allows a defined recording position in the nasal cavity and minimizes variance during the recording of the NMP.

Further investigation of the trigeminal chemoreception of nicotine in humans and rats revealed the existence of specific stereoselective sensory receptors in all probability belonging to the nAchR family (Renner et al, 1998; Thuerauf et al, 1999). Mecamylamine, an acetylcholinereceptor-antagonist, was able to block painful sensations following chemical stimulation of the human tongue and to block responses from the rat's ethmoidal nerve (Alimohammadi and Silver, 2000; Dessirier et al, 1998). Based on these results, it can be hypothesized that mecamylamine blocks nasal trigeminal chemoreception of nicotine in humans by acting as a neuronal nAch-R antagonist at trigeminal sensory receptors. To our knowledge, the detection of nicotine enantiomers in the ultra low concentration range, that is, near the detection threshold, is mediated by the olfactory system. The role of nAchReceptors for the pure olfactory perception of nicotine in the low and ultra low concentration range remains unclear. The local application of mecamylamine might be a suitable tool for testing for the involvement of nAch-Receptors in trigeminal and olfactory chemoreception of nicotine in humans. Thus, we investigated the effects of mecamylamine on the nasal chemoreception of nicotine at olfactory and trigeminal stimulus concentrations in a placebo-controlled double blind study in nonsmokers. The study was designed in order to test the hypothesis (1) that mecamylamine reduces NMPs and trigeminal intensity estimates and increases trigeminal thresholds and (2) that mecamylamine does not influence olfactory intensity estimates and detection thresholds of nicotine. $\mathrm{CO}_{2}$ was used as a trigeminal and $\mathrm{H}_{2} \mathrm{~S}$ as an olfactory control stimulus, supposed to possess transduction mechanisms different from nicotine and insensitive to mecamylamine application.

\section{PARTICIPANTS AND METHODS}

\section{Study Participants}

We evaluated inclusion and exclusion criteria for all subjects by assessing their clinical history, carrying out clinical routine laboratory tests and pregnancy tests, and by conducting a standard physical examination. In order to exclude subjects with relevant smell disturbances from the study, all participants participated in two standard examinations, a subjective olfactory test ('Sniffin Sticks', Hummel et al, 1997) and an acoustical rhinometric test (helps to exclude mechanical obstacles in the nasal cavity, for example, polyposis or septum deviation, Hilberg et al, 1989). Inclusion criteria: non-smokers, age: 18-45 years, body mass index $<30 \mathrm{~kg} / \mathrm{m}^{2}$; exclusion criteria (confirmed by our local ethical committee): relevant smell deficits, mental illnesses (DSM-IV classification), relevant physical diseases, pregnancy and lactancy, intake of other medications except contraceptives, obesity Grade II and higher (body mass index: $>30 \mathrm{~kg} / \mathrm{m}^{2}$ ), age $>45$ years, $<18$ years, 'regular' consumption of cigarettes, participation to other drug studies within the last 30 days, contraindications against mecamylamine (Inversine ${ }^{\circledR}$ ). Subjects fulfilling any exclusion criteria were excluded from the study and subjects fulfilling none of the exclusion but all of the inclusion criteria were included in the study. Written informed consent was signed if all inclusion and no exclusion criteria were fulfilled. In all, seven male and eight female non-smokers (mean age: $31.6 \pm 6.48$ years, minimum: 24 years, maximum: 43 years, $n=15$ ) were included in and participated in the study. All subjects were informed about the aim of the study and gave their written informed consent prior to their inclusion in the study. Subjects were free to interrupt the testing sessions at any time. The study was performed in accordance with the Declaration of Helsinki of 1975 and was approved by the Ethics Committee of the University of Erlangen-Nürnberg. No side effects of the medication were noticed after nasal application of placebo/mecamylamine during the study.

\section{Study Design}

The placebo-controlled double blind study consisted of two experiments. Each experiment consisted of two identical testing sessions representing the mecamylamine- and the placebo-condition (two-fold crossover within each experiment: testing session-1 and testing session-2). We randomized the beginning with experiment- 1 or experiment- 2 and randomized the beginning with the mecamylamine- or the placebo-condition within both experiments. All testing sessions of both experiments were performed on separate 
days with a time interval of 1 week between each session. Prior to the experiments, subjects participated in a short training session in order to become acquainted with the testing environment and the experimental procedures including the testing questionnaires. During the first experiment (experiment-1), we determined the individual detection and trigeminal thresholds and registered intensity estimates (stimulus intensity) and verbal descriptors (stimulus quality) following stimulation with $S-(-)$ - and $R-(+)$-nicotine at individual olfactory and trigeminal stimulus concentrations and following stimulation with control stimuli $\left(\mathrm{CO}_{2}, \mathrm{H}_{2} \mathrm{~S}\right)$. The individual olfactory and trigeminal stimulus concentrations of nicotine were defined in order to elicit an 'odorous' (individual olfactory stimulus concentration) and a 'burning' sensation (individual trigeminal stimulus concentration/low trigeminal stimulus condition). The testing was performed before and after local application of mecamylamine or saline (testing session-1 or testing sessions-2). During the second experiment (experiment-2), we recorded NMPs and registered intensity estimates following trigeminal stimulation with a set stimulus concentration of nicotine enantiomers and following control stimulation with $\mathrm{CO}_{2}$. A high trigeminal stimulus concentration of nicotine was used in order to elicit an 'odorous', a 'burning', and a 'stinging' sensation (set trigeminal stimulus concentration/high trigeminal stimulus condition). The testing was performed before and after local application of mecamylamine or saline (testing session-1 or testing session-2). During both experiments, subjects and experimenter were blind to medication, that is, mecamylamine or saline.

Experiment-1. At the beginning of experiment-1, we determined detection and trigeminal thresholds in a threshold testing session. This procedure also helped to define the individual olfactory and trigeminal suprathreshold stimulus concentrations used in experiment- 1 . Three olfactory and three trigeminal $S-(-)$ - and $R-(+)$-nicotine stimuli and control stimuli $\left(\mathrm{CO}_{2}: 70 \% \mathrm{v} / \mathrm{v} \mathrm{CO}_{2}, n=3\right.$ and $\mathrm{H}_{2} \mathrm{~S}: 7 \mathrm{ppm}, n=3$ ) were applied before and after local application of mecamylamine or saline in a randomized order (stimulus concentrations: individual olfactory and trigeminal stimulus concentration, stimulus duration: $250 \mathrm{~ms}$, interstimulus interval: $120 \mathrm{~s})$. Mecamylamine $(0.225 \mathrm{mg}$, volume: $0.3 \mathrm{ml})$ or saline (volume: $0.3 \mathrm{ml})$ was applied locally to the nasal cavity by means of a nasal spray. Following each stimulation with the test substances, we registered olfactory ('odorous' sensation) and trigeminal intensity estimates ('burning' sensation) via computer monitor and assessed verbal descriptors by means of Dravnieks's (odor quality Dravnieks et al, 1984) and McGill's (pain quality Stein and Mendl, 1988) questionnaires. At the end of experiment-1, we determined detection and trigeminal thresholds in a second threshold testing session in order to assess the effect of mecamylamine vs placebo.

Experiment-2. Five $S$ - $(-)$-, five $R-(+)$-nicotine stimuli, and five $\mathrm{CO}_{2}$-control stimuli (stimulus concentration: $180 \mathrm{ng} / \mathrm{ml}$ $S-(-)-/ R-(+)$-nicotine, $70 \% \mathrm{v} / \mathrm{v} \mathrm{CO}_{2}$, stimulus duration: $250 \mathrm{~ms}$, interstimulus interval: $90 \mathrm{~s}$ ) were applied before and after local application of mecamylamine or saline. All NMPs were recorded under endoscopic control. Mecamylamine $(0.225 \mathrm{mg}$, volume: $0.3 \mathrm{ml})$ or saline (volume: $0.3 \mathrm{ml})$ was applied locally to the nasal cavity by means of a nasal spray. In addition, we registered the olfactory ('odorous' sensation) and the trigeminal intensity estimates ('burning' and 'stinging' sensation) of all perceived stimuli via computer monitor. NMP amplitudes and latencies were determined after offline averaging and submitted to statistical analysis.

\section{Chemical Stimulation}

An olfactometer was used to allow the precise presentation of chemical stimuli $\left(R(+)\right.$ - and $\mathrm{S}(-)$-nicotine, $\left.\mathrm{CO}_{2}, \mathrm{H}_{2} \mathrm{~S}\right)$ without simultaneous activation of mechano- or thermoreceptors in the nasal mucosa of the subjects (constant flow rate: $140 \mathrm{ml} / \mathrm{s}$, humidity: $80 \%$, temperature: $37^{\circ} \mathrm{C}$, stimulus duration, $250 \mathrm{~ms}$, Kobal, 1985). Stimulus nicotine concentrations were determined by online GC-UV detection and offline sampling (concentrations $<2 \mathrm{ng} / \mathrm{ml}$ ) and subsequent GC analysis (Thuerauf et al, 1999). Prior to the measurements, subjects were comfortably installed in a measuring chamber with electronically controlled air circulation. The olfactometer was free of additional chemicals/odorants. Subjects were instructed not to use perfume or to eat foods that emit a strong odor (eg, garlic) prior to the testing session. The nitrogen gas stream containing $R-(+)$ - or $S$-(-)-nicotine continuously generated inside the olfactometer was eliminated during the interstimulus interval by an external sucking device connected to the olfactometer. This procedure prevented contamination of the measuring chamber by outside odors.

Test substances. Optically and chemically pure $R-(+)$-and $S-(-)$-nicotine were produced with the same method by Dr C Mark, Chemisches Laboratorium Worms, Germany. Distilled $R-(+)$-nicotine was produced from $S$ - $(-)$-nicotine (Aldrich, chemical purity 98\%) by racemization and subsequent separation of the $R-(+)$-isomer by crystallization with $R, R$-tartaric acid and di-p-toloyl-S, $S$-tartaric acid. Distilled $S-(-)$-nicotine was produced from $S-(-)$ nicotine (Aldrich, chemical purity $98 \%$ ) by racemization and subsequent separation of the $S-(-)$-isomer by crystallization with $S, S$-tartaric acid and di-p-toloyl- $R, R$-tartaric acid. The distilled $R-(+)$ - and $S-(-)$-nicotine base (colorless liquid) were stored in glass tubes in a nitrogen atmosphere $\left(-60 \mathrm{C}^{\circ}\right)$ until the experiments were started. For the experiments, only the undiluted pure nicotine base of $R-(+)$ - and $S-(-)$-nicotine was used. The optical purity of both enantiomers was greater than $99 \%$. For details of the determination of stimulus concentrations, see Thuerauf et al $(1995,1999)$. For detailed description of the stimulating procedures and substances used in the experiments, see Thuerauf et al (1999, 2000).

\section{Threshold Determination}

At the beginning and end of the testing sessions of experiment-1, we determined the detection and the trigeminal thresholds within a testing range of $0-180 \mathrm{ng} /$ $\mathrm{ml} R-(+)-/ S-(-)$-nicotine employing the method introduced by Wysocki and Beauchamp (1984) (see also Eccles et al, 1989; Zattore and Jones-Gotman, 1990; Hummel et al, 
1992a). Each threshold determination started with the threshold determination of detection, followed by the threshold determinations of the 'burning' and 'stinging' sensations (trigeminal sensations). For orientation, an ascending sequence of $R-(+)-/ S-(-)$-nicotine stimuli was carried out with increases of $3 \mathrm{ng} / \mathrm{ml} R-(+)-/ S-(-)$-nicotine until a stimulus was detected and with increases of $18 \mathrm{ng} / \mathrm{ml}$ $R-(+)-/ S-(-)$-nicotine until a 'burning' and a subsequent 'stinging' sensation were perceived. For threshold determination, ascending stimulus series were executed with a starting point clearly below the individual thresholds of each subject. For each concentration step within the series, two blanks and one stimulus (triple forced choice) were applied in random order. The concentration steps within the series were $0.4 \mathrm{ng} / \mathrm{ml} R-(+)-/ S-(-)$-nicotine for measuring the detection thresholds and $2 \mathrm{ng} / \mathrm{ml} R-(+)-/ S-(-)-$ nicotine for measuring the thresholds of the 'burning' and 'stinging' sensations. A series ended, when a clear sensation could be reported by the subject in three successive stimulus $v s$ blank trials. The lowest value of the three was designated as an estimate of the subject's threshold. For the determination of the detection threshold, a sensation different from blank was requested. Additionally, the subjects' verbal descriptions of the perceived stimuli at the threshold value were documented. For the determination of the trigeminal thresholds, a clear sensation was defined as a clear 'burning' or 'stinging' sensation depending on the threshold to be determined using this procedures. The stimulus duration was $250 \mathrm{~ms}$ in all testing sessions. The interstimulus interval was $1 \mathrm{~min} 30 \mathrm{~s}$.

\section{Individual Olfactory and Trigeminal Nicotine Stimulus Concentrations}

During experiment-1, we applied nicotine stimuli with individual olfactory and trigeminal stimulus concentrations. We defined the individual olfactory and the trigeminal stimulus concentration based on the thresholds determined at the beginning of the testing sessions of experiment-1. In order to define the olfactory stimulus, the difference between the individual detection and the trigeminal thresholds ('burning' sensation) of $S$-(-)-nicotine was calculated, and $40 \%$ of this difference was added to the individual detection threshold value. The trigeminal stimulus was defined as the $30 \%$ value above the individual trigeminal threshold ('burning' sensation) of $S$-(-)-nicotine. We limited the stimulus concentration range to $0-180 \mathrm{ng} / \mathrm{ml}$ in order to exclude the adsorption of amounts of nicotine during the testing sessions which can lead to symptoms of nicotine poisoning. It is well known that nicotine can be adsorbed from the nasal and the buccal mucosa and from the skin. We defined the threshold concentration as being ' $>180 \mathrm{ng} / \mathrm{ml}$ ' if 'burning'or 'stinging' sensations could not be elicited.

\section{Verbal Descriptors}

For the subjective description of the stimuli applied in experiment-1, two questionnaires were used: one for odor (Dravnieks's questionnaires) and one for pain (McGill's pain questionnaires). Subjects used the Dravnieks's questionnaires (olfactory concentrations) and the McGill's pain questionnaires (trigeminal concentrations) to describe the nicotine stimulus before and after local application of mecamylamine or saline.

\section{Intensity Estimates of Individual Olfactory and Trigeminal Nicotine Stimuli}

In experiment-1, subjects estimated at individual olfactory concentrations the intensity of the 'odorous' sensation and at individual trigeminal concentrations the intensity of the 'burning' and of the 'odorous' sensation before and after local application of mecamylamine or saline. The subjects estimated the perceived intensity of each stimulus by means of a visual analog scale displayed on video monitor. In addition, the intensity estimates of the olfactory $\left(\mathrm{H}_{2} \mathrm{~S}\right.$, 'odorous' sensation) and the trigeminal control stimuli $\left(\mathrm{CO}_{2}\right.$, 'stinging' and 'burning' sensation) were registered. For statistical evaluation, the individual subjects' ratings were averaged separately for each stimulus category.

\section{Intensity Estimates of Nicotine Stimuli with a Set Stimulus Concentration}

In experiment-2, we registered the intensity estimates of the 'odorous', the 'burning', and the 'stinging' sensation following stimulation with a set stimulus concentration of $180 \mathrm{ng} / \mathrm{ml} R-(+)$ - or $S-(-)$-nicotine and we registered the intensity estimates of the 'burning' and of the 'stinging' sensation following control stimulation with $\mathrm{CO}_{2}$. For statistical evaluation, the individual subjects' ratings were averaged separately for each stimulus category.

\section{NMP Recordings}

In experiment-2, the NMP was recorded from the right side of the nasal septum by means of a tubular electrode filled with $1 \%$ Ringer-agar containing a silver-chlorided silver wire (impedance $1-5 \mathrm{k}$ at $1 \mathrm{kHz}$ in $0.9 \% \mathrm{NaCl}$ ). A silverchlorided silver EEG electrode was attached to the bridge of the nose and served as reference. The recording electrode was placed under endoscopic control at the septal wall along the line between bony and cartilaginous parts of the nose (distance from the tip of the electrode to the left nostril: $2.5-$ $5 \mathrm{~cm}$ ). Recording site was selected to three major criteria (1) accessibility, (2) low signal to noise ratio of the NMP, and (3) no or low stimulus induced positive artefact preceding the pain-related negativity of the NMP (Hummel et al, 1996) (for the recording positions of the NMPs used in our experiments, see Figure 1). During positioning the testing of up to three electrode placements within the described recording region was allowed in order to optimize the recording criteria. After final placement and before the beginning of the testing series, the electrode was fixed by adjustable clips mounted on a frame similar to lensless glasses. The NMP signals were recorded by means of DC amplifiers (Toennies, GER; band pass DC-30 Hz). After analog-to-digital conversion (sampling rate $62.5 \mathrm{~Hz}$; CED 1401, UK), records of $32768 \mathrm{~ms}$ were obtained. Eye blinks were monitored by means of an EEG electrode located above the left eye and referenced to linked earlobes. Records that might have been affected by movements or eye blinks were excluded from further analysis. For the analysis of the 


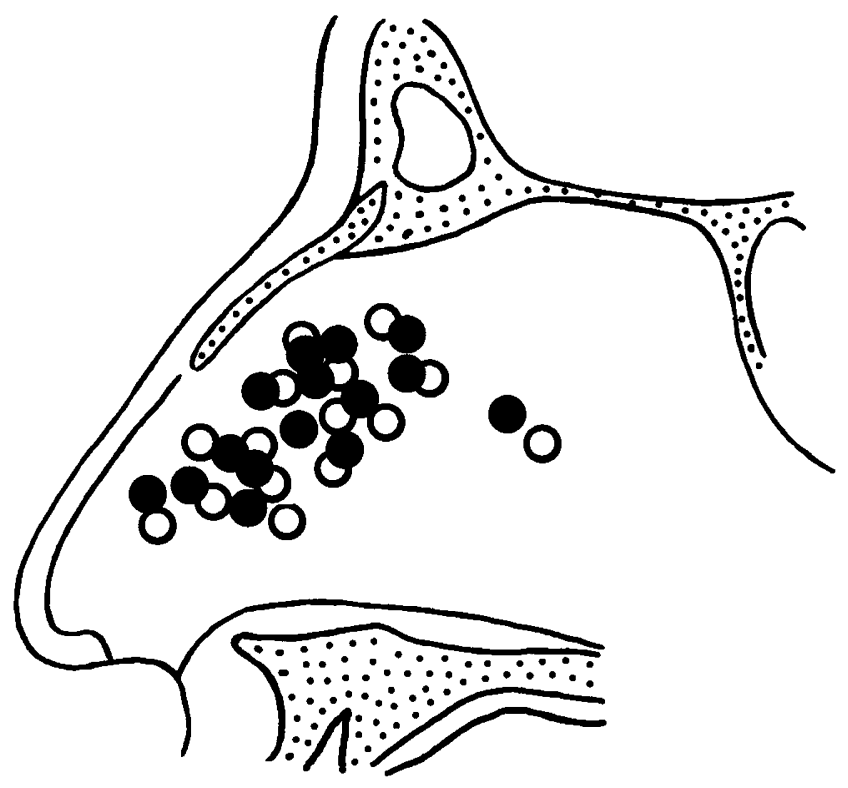

Figure I Schematic drawing of the recording positions of the NMPs (negative mucosal potentials) within the nasal cavity used during experiment-2. Black solid circles: Recording positions before and after $0.225 \mathrm{mg}$ mecamylamine. White circles: Recording positions before and after saline.

data of experiment-2, the remaining records were averaged separately for the three different stimulus categories $(S-(-)$ nicotine, $R-(+)$-nicotine, and $\left.\mathrm{CO}_{2}\right)$. Subsequently, both the latency to the onset (latency-1) and the latency to the maximum amplitude (latency-2) of the NMP were measured in relation to stimulus onset. In addition, the maximum amplitude was measured.

\section{Statistical Analysis}

Experiment-1. A MANOVA was calculated for repeated measurements on one factor, defined by two treatments (mecamylamine, saline). The MANOVAs were calculated separately for the individual olfactory and the individual trigeminal stimulus concentration. At olfactory stimulus concentrations, the dependent variables were the olfactory intensity estimates of $R-(+)-, S-(-)$-nicotine and $\mathrm{H}_{2} \mathrm{~S}$. At trigeminal stimulus concentrations, the dependent variables were trigeminal and olfactory intensity estimates of $R-(+)$ nicotine and $S-(-)$-nicotine and the trigeminal intensity estimates of $\mathrm{CO}_{2}$. We calculated explorative $t$-tests in order to localize significant differences assessed by MANOVA analysis (see asterisk Figure 2).

The nonparametric (Wilcoxon test) test was applied in order to estimate the effects of medication (mecamylamine, saline) on frequency of olfactory and trigeminal descriptors.

Thresholds. If the subject's thresholds could not be determined in this concentration range, we set the thresholds at $>180 \mathrm{ng} / \mathrm{ml}$. Using this procedure, we received for such cases only estimates $(>180 \mathrm{ng} / \mathrm{ml})$ of the thresholds. The subsequent statistical analysis was based on ranking positions and we used the parameter free Wilcoxon Signed Rank Test for the analysis of differences between the
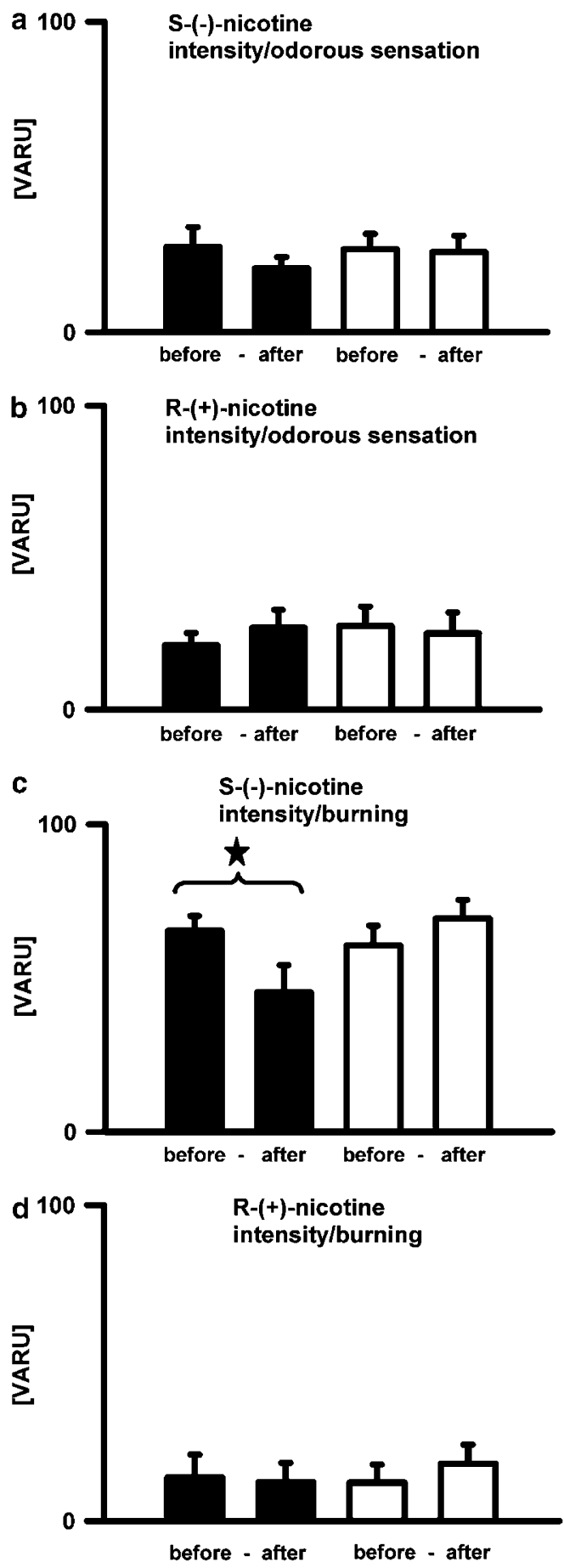

Figure 2 Means and standard errors of means of olfactory (odorous sensation) and trigeminal (burning sensation) intensity estimates after stimulation with individual olfactory $(a, b)$ and individual trigeminal $(c, d)$ nicotine concentrations. Mean value of individual olfactory stimulus concentration: $13.7 \pm 7.1 \mathrm{ng} / \mathrm{ml} \mathrm{R}-(+) / S-(-)$-nicotine $(n=30)$. Mean value of individual trigeminal stimulus concentration: $34.5 \pm 16.5 \mathrm{ng} / \mathrm{ml} \mathrm{R}-(+) / \mathrm{S}$ $(-)$-nicotine $(n=30)$. Black columns: Intensity estimates before and after $0.225 \mathrm{mg}$ mecamylamine $(n=15)$. White columns: Intensity estimates before and after saline $(n=15)$. Asterisks indicate statistically significant differences. VARU: visual analog rating unit.

thresholds for the nicotine stereoisomers in order to determine whether the median of the differences between thresholds for $R-(+)$ - and $S-(-)$-nicotine was different 
from zero. This was evaluated separately for detection and for the 'burning' and the 'stinging' sensation.

\section{Experiment-2}

A MANOVA was calculated for repeated measurements on one factor, defined by two treatments (mecamylamine, saline). The MANOVAs were calculated separately for the chemical stimuli $R-(+)$-nicotine, $S-(-)$-nicotine, and $\mathrm{CO}_{2}$. The dependent variables were the amplitude, latency- 1 and latency- 2 of the NMP, and the olfactory and trigeminal intensity estimates of $R-(+)$ - and $S-(-)$-nicotine and the trigeminal intensity estimates of $\mathrm{CO}_{2}$. We calculated explorative $t$-tests in order to localize significant differences assessed by MANOVA analysis (see asterisk Figures 3-5).

All statistical test were two sided. The significance level $\alpha$ was set at 0.05 . A statistical tendency was considered at $p<0.1$.

\section{RESULTS}

\section{Experiment-1/Thresholds}

Detection thresholds. Mecamylamine did not significantly influence the detection thresholds of $R-(+)$ - and $S-(-)$ nicotine (Wilcoxon test, NS, for means and standard deviations, see Table 1). At the detection thresholds of $R-(+)$ - and $S-(-)$-nicotine, 13 out of 15 subjects perceived an 'odorous' sensation. Two subjects noticed the difference to blanks but were not able to describe the perceived stimuli in a more detailed way.

Trigeminal thresholds. S-(-)-nicotine: The thresholds for the 'burning' and for the 'stinging' sensation were significantly higher following application of mecamylamine (Wilcoxon test, 'burning': $p=0.001$, 'stinging': $p<0.01$, for means, standard deviations, and number of subjects with thresholds out of the stimulus concentration range, see Table 1).

$\mathrm{R}-(+)$-nicotine. The increase in the trigeminal thresholds ('burning' and 'stinging' sensation) following application of mecamylamine did not reach statistical significance in the concentration range tested $(0-180 \mathrm{ng} / \mathrm{ml} R-(+)$-nicotine $)$. However, for $R-(+)$-nicotine, 'burning' and 'stinging' sensations were rarely achieved in the applied concentrations range (for means, standard deviations, and number of subjects with thresholds out of the stimulus concentration range, see Table 1 ).

\section{Experiment-1/Individual Olfactory and Trigeminal Stimulus Concentrations}

The mean and standard deviation values of the individual olfactory and trigeminal stimulus concentrations used in experiment-1 were $13.7 \pm 7.1$ and $34.5 \pm 16.5 \mathrm{ng} / \mathrm{ml}(n=30)$. The calculation of the individual olfactory and trigeminal stimulus concentrations was based on thresholds for $S-(-)$ nicotine determined before application of mecamylamine/ saline during testing session- $1(n=15)$ and testing session$2(n=15)$ of experiment-1 (see also 'methods' above). Statistical control analysis revealed no differences between the thresholds determined in testing session-1 and -2 ( $t$-test, NS).

\section{Experiment-1/Verbal Descriptors}

The statistical analysis revealed no consistent and significant effects of mecamylamine (compared to placebo) on verbal descriptors. The most frequent trigeminal descriptors for $S$-(-)-nicotine were 'stinging', 'burning', and 'sharp' (McGill, before/after mecamylamine: 'stinging': 6/2, 'burning': 12/10, 'sharp': 7/3).

Table I Thresholds: Means, Standard Deviations, and Number of Subjects with Thresholds out of the Stimulus Concentration Range of $0-180 \mathrm{ng} / \mathrm{ml}$

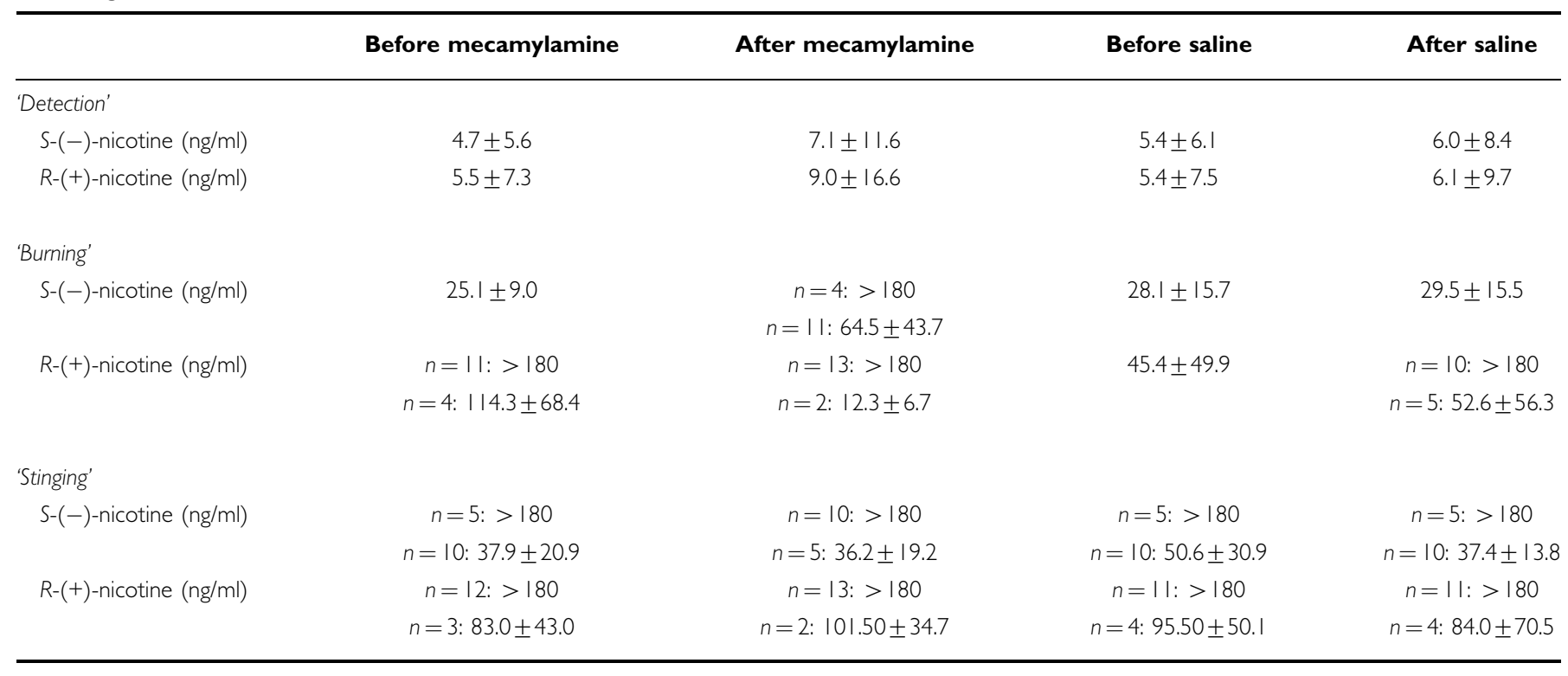




\section{Experiment-1/Intensity Estimates of Individual Olfactory and Trigeminal Nicotine Stimuli}

Olfactory intensity estimates at individual olfactory concentrations. Mecamylamine did not significantly influence the olfactory intensity estimates of $R-(+)$ - and $S-(-)$ nicotine (MANOVA, NS, for means and SEMs (standard error of means), see Figure 2).

Trigeminal Intensity estimates at individual trigeminal concentrations following stimulation with S-(-)-nicotine. Mecamylamine significantly reduced the intensity estimates of the 'burning' sensation (MANOVA, $\mathrm{F}=4.86, p=0.016$, for means and SEMs, see Figure 2).

Trigeminal intensity estimates at individual trigeminal concentrations following stimulation with $\mathrm{R}-(+)$-nicotine. The effect of mecamylamine on the intensity estimates of the 'burning' sensation did not reach statistical significance (MANOVA, NS, for means and SEMs, see Figure 2). For $R-(+)$-nicotine, 'burning' was rarely achieved in the concentrations range tested.

Trigeminal intensity estimates following control stimulation with $\mathrm{CO}_{2}\left(70 \% \mathrm{v} / v \mathrm{CO}_{2}\right)$. Mecamylamine did not significantly reduce the intensity estimates of the 'burning' and of the 'stinging' sensation (MANOVA, NS, for means and standard deviations, see Table 2).

Olfactory intensity estimates at individual trigeminal concentrations following stimulation with S-(-)- and $\mathrm{R}-(+)$-nicotine. Mecamylamine did not significantly reduce the intensity estimates of the 'odorous' sensation (MANOVA, NS, for means and standard deviations, see Table 3).

Olfactory intensity estimates following control stimulation with $\mathrm{H}_{2} \mathrm{~S}(7 \mathrm{ppm})$. Mecamylamine did not significantly reduce the intensity estimates of the 'odorous' sensation (MANOVA, NS, for means and standard deviations, see Table 2).

\section{Experiment-2/NMPs}

NMP amplitudes following stimulation with $\mathrm{R}-(+)-$ and $\mathrm{S}-(-)$-nicotine and $\mathrm{CO}_{2}$. Mecamylamine significantly reduced NMP amplitudes of $R-(+)$ - and $S$-(-)-nicotine $(R-(+)$-nicotine: MANOVA, $\mathrm{F}=21.90, p<0.001, S-(-)$ nicotine: MANOVA, $\mathrm{F}=15.80, p<0.001$, for means and SEMs, see Figure 3). Mecamylamine did not significantly reduce NMP amplitudes of the control stimulus $\mathrm{CO}_{2}(70 \%$ v/v $\mathrm{CO}_{2}$, MANOVA, NS, for means and SEMs, see Figure 3). Figure 4 gives a representative single example of an NMP recording following stimulation with $S-(-)$-nicotine and $\mathrm{CO}_{2}$ illustrating the different effect of mecamylamine on both stimuli.

NMP latency-1 following stimulation with $\mathrm{R}-(+)-$, S- $(-)-$ nicotine and $\mathrm{CO}_{2}$. Mecamylamine did not significantly increase the latency-1 for $R-(+)$-nicotine. A statistical tendency of the increase could be observed (MANOVA, $\mathrm{F}=2.65, p=0.089)$. The increase in latency-1 for $S-(-)$ nicotine did not reach statistical significance. A statistical tendency of the increase could be observed (MANOVA, $\mathrm{F}=3.05, p=0.064)$. Mecamylamine did not significantly influence the latency-1 of the control stimulus $\mathrm{CO}_{2}(70 \% \mathrm{v} / \mathrm{v}$ $\mathrm{CO}_{2}$, MANOVA, NS). For means and standard deviations of NMP-latencies-1 of $R-(+)-, S-(-)$-nicotine and $\mathrm{CO}_{2}$, see Table 4.

NMP latency-2 following stimulation with R-(+)-, S-(-)nicotine and $\mathrm{CO}_{2}$. Mecamylamine did not significantly influence the latency-2 of $R-(+)$ - and $S-(-)$-nicotine (MANOVA, NS). Mecamylamine did not significantly influence the latency-2 of the control stimulus $\mathrm{CO}_{2}(70 \%$ $\mathrm{v} / \mathrm{v} \mathrm{CO}_{2}, \mathrm{MANOVA}$, NS). For means and standard deviations of NMP-latencies-1 of $R-(+)-, S-(-)$-nicotine and $\mathrm{CO}_{2}$, see Table 4 .

\section{Experiment-2/Intensity Estimates of Nicotine Stimuli} with a Set Stimulus Concentration

Trigeminal intensity estimates at high $(180 \mathrm{ng} / \mathrm{ml} \mathrm{R}-(+)-/$ S-(-)-nicotine) trigeminal stimulus concentration. Mecamyl-

Table 2 Means and Standard Deviations of the Intensity Estimates of Olfactory $\left(\mathrm{H}_{2} \mathrm{~S}\right)$ and Trigeminal $\left(\mathrm{CO}_{2}\right)$ Control Stimuli

\begin{tabular}{lcrr}
\hline Stimulus & Before mecamylamine & After mecamylamine & Before saline \\
\hline $\mathrm{H}_{2} \mathrm{~S}$ (VARU) 'Odor' & $62.9 \pm 26.1$ & $59.1 \pm 33.4$ & $69.7 \pm 26.1$ \\
$\mathrm{CO}_{2}$ (VARU) 'Burning' & $54.3 \pm 38.3$ & $44.6 \pm 39.7$ & $60.4 \pm 35.4$ \\
$\mathrm{CO}_{2}$ (VARU) 'Stinging' & $78.3 \pm 26.1$ & $80.4 \pm 26.4$ & $75.0 \pm 27.9$ \\
\hline
\end{tabular}

VARU: visual analog rating unit.

Table 3 Means and Standard Deviations of Olfactory Intensity Estimates Following Stimulation with Individual Trigeminal Nicotine Concentrations

\begin{tabular}{lcrr}
\hline Stimulus & Before mecamylamine & After mecamylamine & Before saline \\
\hline S-(-)-nicotine (VARU) & $44.4 \pm 31.1$ & $28.5 \pm 30.3$ & $34.8 \pm 22.0$ \\
R-(+)-nicotine (VARU) & $32.7 \pm 18.7$ & $33.2 \pm 25.7$ & $39.3 \pm 27.9$
\end{tabular}

VARU: visual analog rating unit. 
amine significantly reduced the intensity estimates of the 'burning' and of the 'stinging' sensation following stimulation with $S-(-)$ - and $R-(+)$-nicotine $(S-(-)$-nicotine: MANOVA
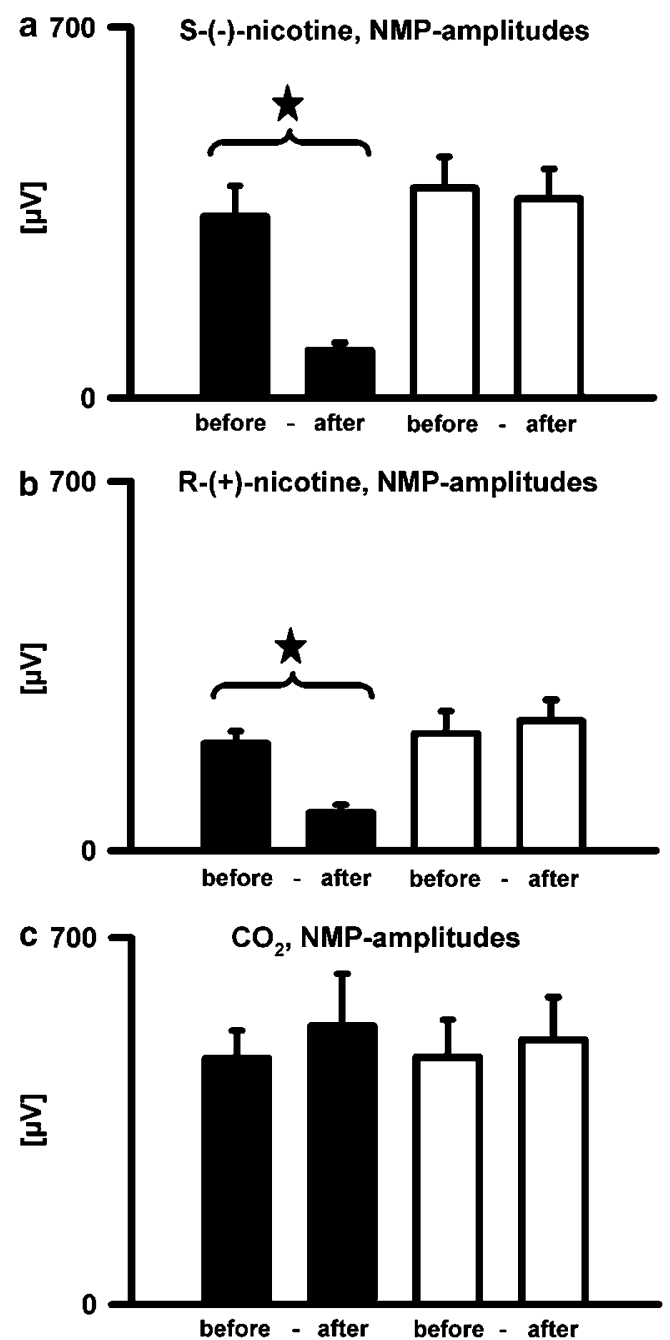

Figure 3 Means and standard errors of means of NMP amplitudes recorded after stimulation with (a) $180 \mathrm{ng} / \mathrm{ml} \mathrm{S}-(-)$-nicotine, (b) $180 \mathrm{ng} / \mathrm{ml}$ R-(+ )-nicotine, (c) $70 \% \mathrm{v} / \mathrm{v} \mathrm{CO}_{2}$. Black columns: NMP amplitudes before and after $0.225 \mathrm{mg}$ mecamylamine $(n=15)$. White columns: NMP amplitudes before and after saline $(n=15)$. Asterisks indicate statistically significant differences. NMP: negative mucosal potential. 'burning', $\mathrm{F}=36.92, p<0.001$, MANOVA 'stinging', $\mathrm{F}=22.98$, $p<0.001, \quad R-(+)$-nicotine: MANOVA 'burning', $\mathrm{F}=9.98$, $p=0.001$, MANOVA 'stinging', $\mathrm{F}=6.31, p=0.006$, for means and SEMs, see Figures 5 and 6). Mecamylamine did not significantly influence the intensity estimates of the 'burning'
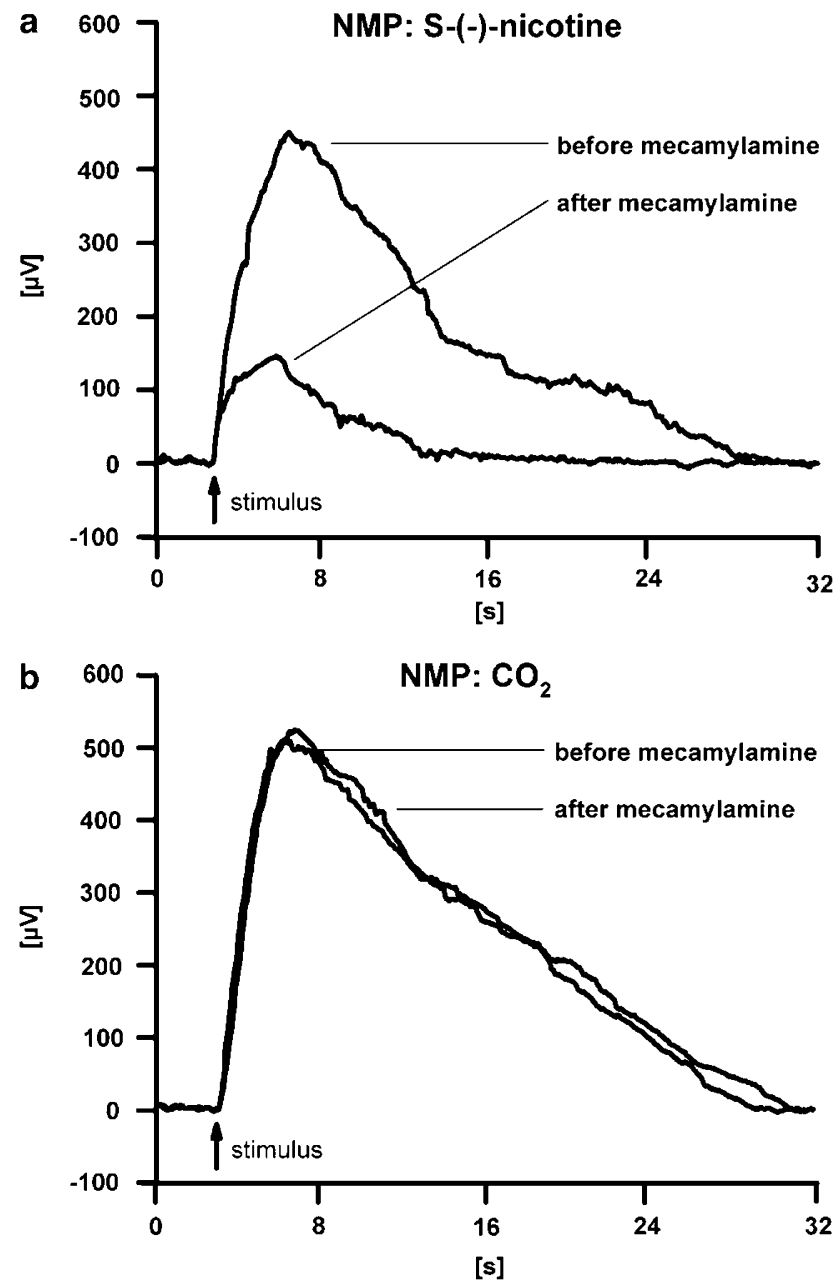

Figure 4 NMP recordings of a single subject following stimulation with (a) $180 \mathrm{ng} / \mathrm{ml} \mathrm{S}$-(-)-nicotine and (b) $70 \% \mathrm{v} / \mathrm{v} \mathrm{CO}_{2}$ before and after $0.225 \mathrm{mg}$ mecamylamine. Averaged responses ( $n=5$ for both stimulus categories), stimulus duration: $250 \mathrm{~ms}$, interstimulus interval: $90 \mathrm{~s}$.

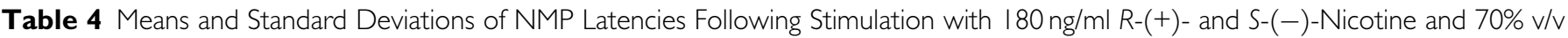
$\mathrm{CO}_{2}$

\begin{tabular}{|c|c|c|c|c|}
\hline & Before mecamylamine & After mecamylamine & Before saline & After saline \\
\hline \multicolumn{5}{|l|}{ Latency-I (ms) } \\
\hline S-(-)-nicotine & $504 \pm 95$ & $633 \pm 197$ & $490 \pm 119$ & $499 \pm 101$ \\
\hline$R$-(-)-nicotine & $518 \pm 67$ & $739 \pm 440$ & $491 \pm 85$ & $513 \pm 145$ \\
\hline $\mathrm{CO}_{2}$ & $505 \pm 87$ & $473 \pm 51$ & $469 \pm 131$ & $485 \pm 137$ \\
\hline$R-(+)$-nicotine & $3645 \pm 2073$ & $3291 \pm 1916$ & $3452 \pm 1930$ & $3346 \pm 2767$ \\
\hline $\mathrm{CO}_{2}$ & $4220 \pm 1243$ & $4180 \pm 1729$ & $4090 \pm 1442$ & $4005 \pm|46|$ \\
\hline
\end{tabular}


and 'stinging' sensation following stimulation with $\mathrm{CO}_{2}(70 \%$ $\mathrm{v} / \mathrm{v} \mathrm{CO}_{2}$, MANOVA 'burning', NS, MANOVA 'stinging', NS, for means and SEMs, see Figures 5 and 6).

Olfactory intensity estimates at high $(180 \mathrm{ng} / \mathrm{ml} \mathrm{R}-(+)-/$ $\mathrm{S}-(-)$-nicotine) trigeminal stimulus concentration. Mecamylamine did not significantly influence the olfactory
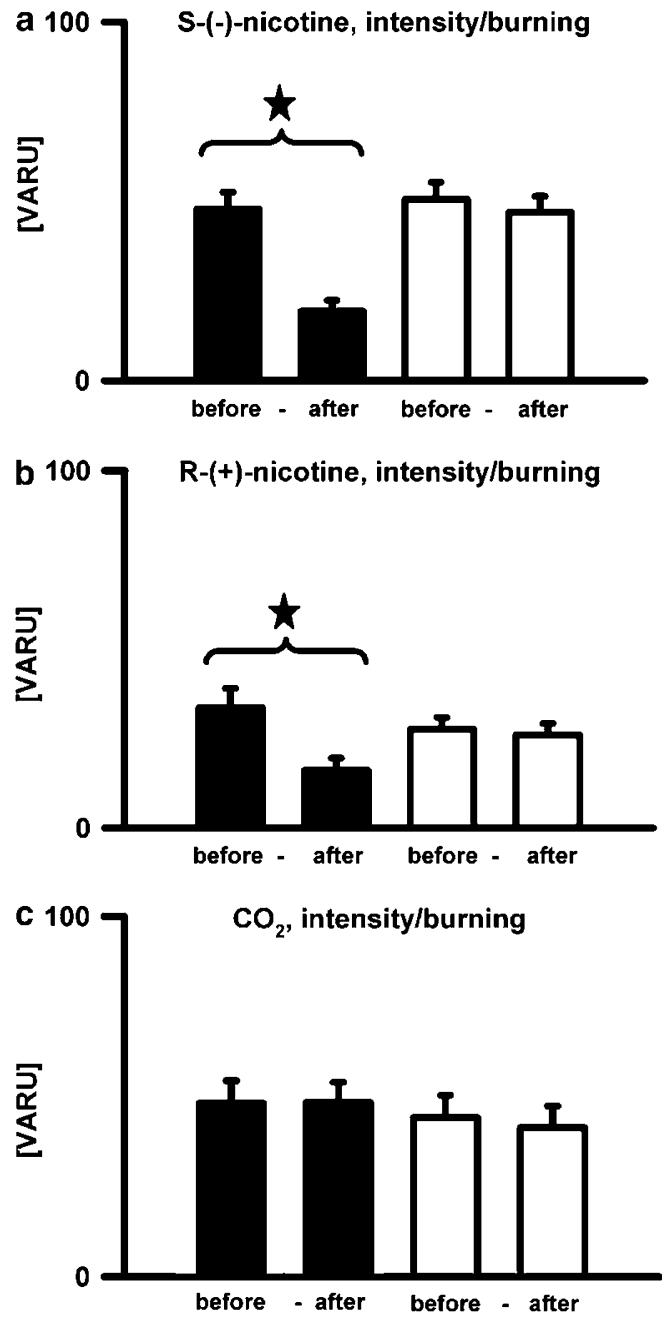

Figure 5 Means and standard errors of means of the intensity estimates for the burning sensation after stimulation with (a) $180 \mathrm{ng} / \mathrm{ml} \mathrm{S}-(-)$ nicotine, (b) $180 \mathrm{ng} / \mathrm{ml} \mathrm{R}-(+)$-nicotine, (c) $70 \% \mathrm{v} / \mathrm{v} \mathrm{CO}$. Black columns: Intensity estimates before and after $0.225 \mathrm{mg}$ mecamylamine $(n=15)$. White columns: Intensity estimates before and after saline $(n=15)$. Asterisks indicate statistically significant differences. VARU: visual analog rating unit intensity estimates of $R-(+)$ - and $S$ - $(-)$-nicotine (MANO$\mathrm{VA}, \mathrm{NS}$, for means and standard deviations, see Table 5).

\section{DISCUSSION}

At high trigeminal stimulus concentrations, mecamylamine significantly reduced the trigeminal intensity ratings
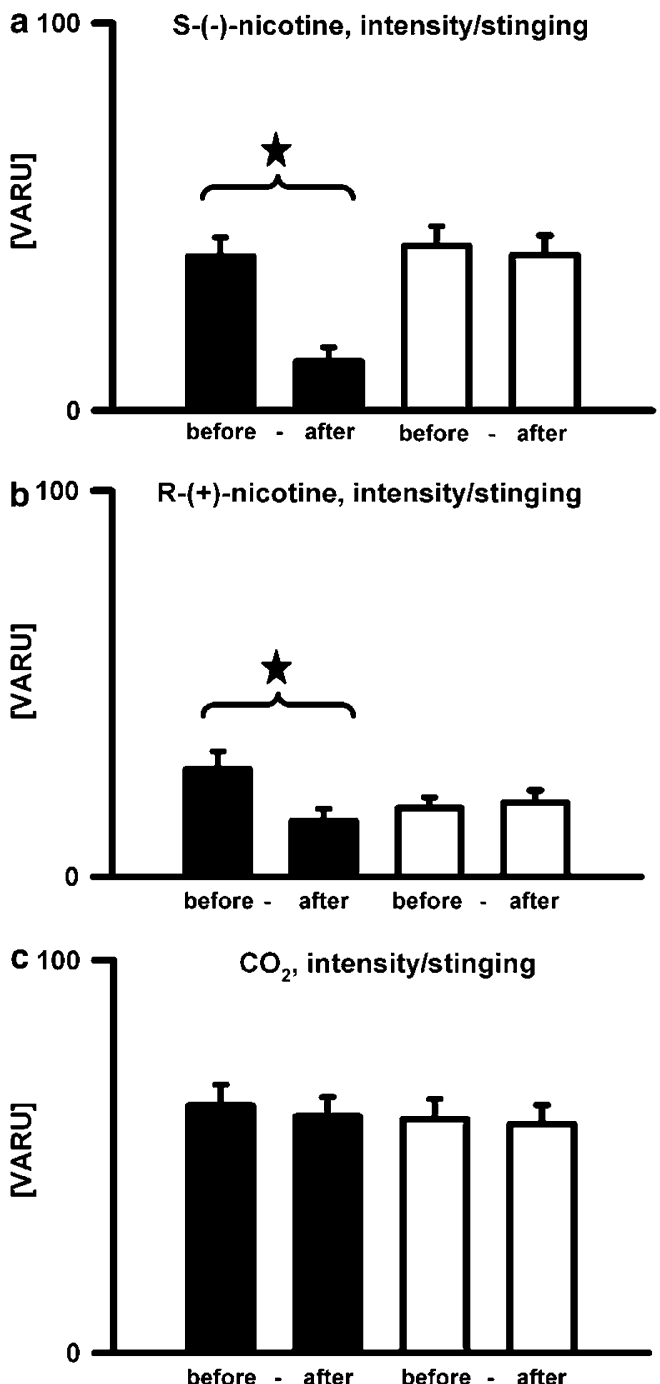

Figure 6 Means and standard errors of means of the intensity estimates for the stinging sensation after stimulation with (a) $180 \mathrm{ng} / \mathrm{ml} \mathrm{S}$-(-)-nicotine (b) $180 \mathrm{ng} / \mathrm{ml} \mathrm{R-(+)-nicotine} \mathrm{(c)} 70 \% \mathrm{v} / \mathrm{v} \mathrm{CO}_{2}$. Black columns: Intensity estimates before and after $0.225 \mathrm{mg}$ mecamylamine $(n=15)$. White columns: Intensity estimates before and after saline $(n=15)$. Asterisks indicate statistically significant differences. VARU: visual analog rating unit.

Table 5 Means and Standard Deviations of the Olfactory Intensity Estimates Following Stimulation with a Set Trigeminal Nicotine Concentration ( $180 \mathrm{ng} / \mathrm{ml})$

\begin{tabular}{lcrr}
\hline Stimulus & Before mecamylamine & After mecamylamine & Before saline \\
\hline S-(-)-nicotine (VARU) & $32.3 \pm 18.1$ & $30.2 \pm 19.7$ & $39.6 \pm 21.0$ \\
R-(+)-nicotine (VARU) & $35.9 \pm 19.7$ & $29.2 \pm 22.0$ & $42.4 \pm 22.4$
\end{tabular}

VARU: visual analog rating unit. 
('burning' and 'stinging') and the NMPs registered following stimulation with $R-(+)$ - and $S$ - $(-)$-nicotine. At lower trigeminal stimulus concentrations as used for assessing the 'burning' sensations in experiment-1, mecamylamine significantly reduced the trigeminal intensity estimates of $S-(-)$-nicotine. The reduction observed for $R-(+)$-nicotine did not reach statistical significance, which can easily be explained by the weaker stimulus strength of $R-(+)$ nicotine and the resulting lower signal to noise ratio of $R-(+)$-nicotine's trigeminal intensity estimates. Mecamylamine significantly increased the trigeminal thresholds of $S$-(-)-nicotine for the 'burning' and for the 'stinging' sensation. The increase observed for $R-(+)$-nicotine did not reach statistical significance, but 'burning' and 'stinging' sensations were rarely achieved before medication because of $R$ - $(+)$-nicotine's weak trigeminal stimulus strength and because of ethical limitations with respect to stimulus concentrations. Microneurographic studies in humans demonstrated that activation of a single $\mathrm{C}$-fiber could evoke a 'burning' sensation and activation of a single A $\delta$-fiber could evoke a 'stinging' sensation (for review, see Handwerker and Kobal, 1993). In view of these microneurographic results, our psychophysical data indicate stereoselective activation of both subgroups of nociceptive fibers following stimulation with nicotine. The fact that at high nicotine concentrations mecamylamine significantly reduced all trigeminal intensity estimates of $R-(+)$ - and $S$-(-)-nicotine indicates that mecamylamine-sensitive nicotinic receptors are present on $\mathrm{C}$ - and $\mathrm{A} \delta$-fibers. The higher threshold for the 'stinging' than for the 'burning' sensation observed in this and in earlier studies point to different stereoselective receptors on $\mathrm{C}$ - and $\mathrm{A} \delta$-fibers. We found no significant effects of mecamylamine on stimulus quality, that is, on verbal descriptors following stimulation with nicotine. One explanation might be the fact that mecamylamine acting as a broad-spectrum noncompetitive antagonist of nAch-Receptors (Papke et al, 2001; Woodruff-Pak, 2003) reduces but does not extinguish trigeminal sensations. Thus, it leads to a decrease in trigeminal intensity estimates but not to a clear change in stimulus quality. Mecamylamine significantly reduced NMPs elicited by chemical stimulation with $S-(-)$ - and $R-(+)$-nicotine but did not influence NMPs elicited by chemical stimulation with $\mathrm{CO}_{2}$. The strongest effect of mecamylamine was found for the NMP's amplitudes, which significantly decreased following application of the drug. The reduction of NMPs coincided with a significant reduction of the trigeminal intensity estimates registered during NMP recordings. A statistical tendency of an increase in latency-1 could be observed for $R-(+)$ - and $S$ - $(-)$-nicotine. Generally, our findings for the effects of mecamylamine on NMP parameters and intensity estimates are in accordance with earlier results form our laboratory demonstrating that amplitudes are more sensitive parameters for small changes in stimulus strength than the NMP's onset (latency-1) (Thuerauf et al, 2002). Both, the reduction of NMPs and the reduction of trigeminal intensity estimates following application of mecamylamine confirm our hypothesis that the trigeminal nasal chemoreception of nicotine enantiomers is mediated by specific stereoselective nasal receptors belonging to the nAchR family (Renner et al, 1998; Thuerauf et al, 1999). Our results are in line with the observations that mecamylamine is able to block painful sensations following chemical stimulation of the human tongue and to block responses from the rat's ethmoidal nerve (Alimohammadi and Silver, 2000; Dessirier et al, 1998). Mecamylamine did not significantly influence the intensity ratings and NMPs following stimulation with $\mathrm{CO}_{2}$ and the intensity estimates following stimulation with $\mathrm{H}_{2} \mathrm{~S}$. These results confirm that nAchReceptors are not involved in the transduction mechanism of $\mathrm{H}_{2} \mathrm{~S}$ and $\mathrm{CO}_{2}$ and recommend both stimuli as sensory control stimuli insensitive to nAchR antagonists in sensory models.

Earlier studies in humans and rats characterized the NMP as a specific and sensitive peripherally nociceptive correlate of trigeminal activation. Our successful blocking experiments with mecamylamine extend these findings in the way that NMP recordings are also suitable for quantifying specific pharmacological alterations of trigeminal chemoreception in humans. NMP recordings combined with the application of specific antagonists represent a specific and objective tool for testing for specific receptor subsets and/or specific transduction mechanisms in trigeminal chemosensory models. These possibilities could help to gain new insights into the trigeminal chemoreception of irritants and odorants with a trigeminal sensory component.

Earlier studies from our laboratory provided evidence for a conditioning mechanism between the trigeminal sensory stimulus and the pleasant pharmacological actions of $S-(-)$ nicotine (Thuerauf et al, 2000; Hummel et al, 1992a). Conditioning processes are considered to represent a key mechanism in maintaining drug dependency (Grusser et al, 2000). Clinicians frequently report the importance of sensory key stimuli causing relapse in addictive patients, for example, the smell of beer when perceived by former alcoholics. Thus, experimental tools like sensory antagonization with mecamylamine aiming at influencing conditioning processes in smokers are of paramount clinical importance. A study assessing the influence of mecamylamine on the hedonic estimates of $S$ - $(-)$-nicotine in smokers and non-smokers is still lacking.

Nicotine replacement is considered to be a standard therapy during smoking cessation (American Psychiatric Association, 1996). One improvement in nicotine replacement aims at substituting not only systemic nicotine but also all sensory input produced by $S-(-)$-nicotine, which is the natural nicotine enantiomer in tobacco (Westman et al, 1995, 1996; Kornitzer et al, 1995). Chewing gums, nasal sprays, and inhalers containing $S$ - $(-)$-nicotine have proved to be effective (Cepeda Benito, 1993; Silagy et al, 1994; Sutherland et al, 1992; Tonnesen et al, 1993). Our results indicate that the activation of sensory trigeminal nAchReceptors is specifically required to replace nicotine's sensory input during smoking cessation. Our sensory experimental model presented here could also help to test for suitable compounds for the sensory replacement of nicotine with psychophysical and electrophysiological (NMPs) means.

Mecamylamine did not significantly influence the olfactory intensity estimates of nicotine following stimulation with olfactory and trigeminal stimulus concentrations characterizing the olfactory system as being insensitive to mecamylamine. At the threshold concentrations of 
detection, 13 out of 15 subjects reported an 'odorous' sensation and only two subjects were not able to characterize a perceived sensation different from blanks in a more detailed manner. This observation indicates that the perception of nicotine enantiomers at threshold concentrations of detection is mediated by the olfactory system. However, a recent study in rats and mice discovered a new type of chemosensory cells - the solitary chemoreceptor cell - in the nasal mucosa expressing T2R 'bitter-taste' receptors and $\alpha$-gustducin, a G-protein involved in chemosensory transduction (Finger et al, 2003). These $\alpha$ gustducin-positive cells showed the characteristics of receptor cells, that is, microvilli at apical processes extending into the nasal lumen. Dual-label immunocytochemistry and electron microscopic analysis showed contacts between solitary chemoreceptor cells and nerve fibers of the trigeminal nerve. Further functional experiments demonstrated that bitter substances applied to the nasal epithelium activate the trigeminal nerve and evoke changes in the respiratory rate (Finger et al, 2003). Based on these results a role of solitary chemoreceptor cells in protective reflexes such as sneezing, coughing, and apnea has been proposed. The ongoing chemoreceptive investigation into the different functions and into different subsets of solitary chemoreceptor cells has not been completed yet. At the moment, a role of solitary chemoreceptor cells in the perception of nicotine even at threshold concentrations of detection cannot be ruled out. However, the detection of nicotine by solitary chemoreceptor cells connected to the trigeminal nerve would be likely to be perceived as an 'irritating' sensation and not as an 'odorous' sensation because the trigeminal and olfactory nerve connect to distinct sensory systems within the brain. In our study, mecamylamine did not significantly influence the detection thresholds of $S-(-)$-and $R-(+)$-nicotine. These findings clearly demonstrate that the chemoreception of nicotine at or near the threshold concentration of detection is independent from nAch-Receptors sensitive to mecamylamine. Furthermore, based on (1) the supersensitivity of the olfactory system (Menini et al, 1995), on (2) the reported 'odorous' sensations at threshold concentrations of detection, and on (3) the broad nAch-R antagonism of mecamylamine (Papke et al, 2001) (Woodruff-Pak, 2003), it can be assumed that the chemoreception of nicotine at low and ultralow concentrations is exclusively mediated by binding to receptors of the olfactory receptor family and not by binding to peripheral nAch-Receptors.

\section{ACKNOWLEDGEMENTS}

The research described in this article was supported by Philip Morris USA Inc. (Philip Morris External Research Program/Fellowship). We thank Susanne Schroeder-Thuerauf (Department of Languages for Special Purposes, University of Erlangen-Nürnberg, Germany) and Bruce Bryant (Monell Chemical Senses Center, Philadelphia, PA USA) for helpful suggestions during the preparation of the manuscript and Elfriede Hoh (Department of Psychiatry and Psychotherapy, University of Erlangen-Nürnberg) for assistance during the experiments.

\section{REFERENCES}

Alimohammadi H, Silver WL (2000). Evidence for nicotinic acetylcholine receptors on nasal trigeminal nerve endings of the rat. Chem Senses 25: 61-66.

American Psychiatric Association (1996). Practice guideline for the treatment of patients with nicotine dependence. Am J Psychiatry 153: $1-31$.

Cepeda Benito A (1993). Meta-analytical review of the efficacy of nicotine chewing gum in smoking treatment programs. J Consult Clin Psychol 61: 822-830.

Davies HM, Vaught A (1990). Research Cigarettes. University of Kentucky Printing Services: Kentucky.

Dessirier JM, O'Mahony M, Sieffermann JM, Carstens E (1998). Mecamylamine inhibits nicotine but not capsaicin irritation on the tongue: psychophysical evidence that nicotine and capsaicin activate separate molecular receptors. Neurosci Lett 240: 65-68.

Dravnieks A, Masurat T, Lamm RA (1984). Hedonics of odors and odor descriptors. J Air Pollution Control Assoc 34: $752-755$.

Eccles R, Jawad MS, Morris S (1989). Olfactory and trigeminal thresholds and nasal resistance to airflow. Acta Otolaryngol Stockh 108: 268-273.

Finger TE, Bottger B, Hansen A, Anderson KT, Alimohammadi H, Silver WL (2003). Solitary chemoreceptor cells in the nasal cavity serve as sentinels of respiration. Proc Natl Acad Sci USA 100: 8981-8986.

Grusser SM, Heinz A, Flor H (2000). Standardized stimuli to assess drug craving and drug memory in addicts. J Neural Transm 107: 715-720.

Handwerker HO, Kobal G (1993). Psychophysiology of experimentally induced pain. Physiol Rev 73: 639-671.

Hilberg O, Jackson AC, Swift DL, Pedersen OF (1989). Acoustic rhinometry: evaluation of nasal cavity geometry by acoustic reflection. J Appl Physiol 66: 295-303.

Hummel T, Hummel C, Pauli E, Kobal G (1992a). Olfactory discrimination of nicotine-enantiomers by smokers and nonsmokers. Chem Senses 17: 13-21.

Hummel T, Livermore A, Hummel C, Kobal G (1992b). Chemosensory event-related potentials in man: relation to olfactory and painful sensations elicited by nicotine. Electroencephalogr Clin Neurophysiol 84: 192-195.

Hummel T, Schiessl C, Wendler J, Kobal G (1996). Peripheral electrophysiological responses decrease in response to repetitive painful stimulation of the human nasal mucosa. Neurosci Lett 212: $37-40$

Hummel T, Sekinger B, Wolf SR, Pauli E, Kobal G (1997). Sniffin' sticks': olfactory performance assessed by the combined testing of odor identification, odor discrimination and olfactory threshold. Chem Senses 22: 39-52.

Kobal G (1985). Pain-related electrical potentials of the human nasal mucosa elicited by chemical stimulation. Pain 22: 151-163.

Kornitzer M, Boutsen M, Dramaix M, Thijs J, Gustavsson G (1995). Combined use of nicotine patch and gum in smoking cessation: a placebo-controlled clinical trial. Prev Med 24: 41-47.

Laviolette SR, van der Kooy D (2004). The neurobiology of nicotine addiction: bridging the gap from molecules to behaviour. Nat Rev Neurosci 5: 55-65.

Menini A, Picco C, Firestein S (1995). Quantal-like current fluctuations induced by odorants in olfactory receptor cells (see comments). Nature 373: 435-437.

Papke RL, Sanberg PR, Shytle RD (2001). Analysis of mecamylamine stereoisomers on human nicotinic receptor subtypes. J Pharmacol Exp Ther 297: 646-656.

Renner B, Meindorfner F, Kaegler M, Thuerauf N, Barocka A, Kobal G (1998). Discrimination of $R$ - and $S$-nicotine by the trigeminal nerve. Chem Senses 29: 602. 
Silagy C, Mant D, Fowler G, Lodge M (1994). Meta-analysis on efficacy of nicotine replacement therapies in smoking cessation. Lancet 343: 139-142.

Stein C, Mendl G (1988). The German counterpart to McGill Pain Questionnaire. Pain 32: 251-255.

Sutherland G, Stapleton JA, Russell MA, Jarvis MJ, Hajek P, Belcher $M$ et al (1992). Randomised controlled trial of nasal nicotine spray in smoking cessation. Lancet 340: 324-329.

Thuerauf N, Friedel I, Hummel C, Kobal G (1991). The mucosal potential elicited by noxious chemical stimuli with $\mathrm{CO}_{2}$ in rats: is it a peripheral nociceptive event? Neurosci Lett 128: 297-300.

Thuerauf N, Günther M, Pauli E, Kobal G (2002). Sensitivity of the negative mucosal potential to the trigeminal target stimulus $\mathrm{CO}_{2}$. Brain Res 942: 79-86.

Thuerauf N, Hummel T, Kettenmann B, Kobal G (1993). Nociceptive and reflexive responses recorded from the human nasal mucosa. Brain Res 629: 293-299.

Thuerauf N, Kaegler M, Dietz R, Barocka A, Kobal G (1999). Dosedependent stereoselective activation of the trigeminal sensory system by nicotine in man. Psychopharmacology 142: 236-243.

Thuerauf N, Kaegler M, Renner B, Barocka A, Kobal G (2000). Specific sensory detection, discrimination, and Hedonic estimation of nicotine enantiomers in smokers and nonsmokers: are there limitations in replacing the sensory components in nicotine. J Clin Psychopharmacol 20: 472-478.
Thuerauf N, Renner B, Kobal G (1995). Responses recorded from the frog olfactory epithelium after stimulation with $R(+)$ - and S(-)-nicotine. Chem Senses 20: 337-344.

Tonnesen P, Norregaard J, Mikkelsen K, Jorgensen S, Nilsson F (1993). A double-blind trial of a nicotine inhaler for smoking cessation. JAMA 269: 1268-1271.

Walker JC, Kendal-Reed M, Keiger CJ, Bencherif M, Silver WL (1996). Olfactory and trigeminal responses to nicotine. Drug Dev Res 38: 160-168.

Westman EC, Behm FM, Rose JE (1995). Airway sensory replacement combined with nicotine replacement for smoking cessation: a randomized, placebo controlled trial using a citric acid inhaler. Chest 107: 1358-1364.

Westman EC, Behm FM, Rose JE (1996). Dissociating the nicotine and airway sensory effects of smoking. Pharmacol Biochem Behav 53: 309-315.

Woodruff-Pak DS (2003). Mecamylamine reversal by nicotine and by a partial alpha7 nicotinic acetylcholine receptor agonist (GTS-21) in rabbits tested with delay eyeblink classical conditioning. Behav Brain Res 143: 159-167.

Wysocki CJ, Beauchamp GK (1984). Ability to smell androstenone is genetically determined. Proc Natl Acad Sci USA 81: 4899-4902.

Zattore RJ, Jones-Gotman M (1990). Right-nostril advantage for discrimination of odors. Percept Psychophys 47: 526-531. 